\title{
Outcomes of ventriculoperitoneal shunt insertion in Sub-Saharan Africa
}

\author{
Clinical article
}

\author{
Esther Gathura, M.B.B.S., Dan Poenaru, M.D., Richard Bransford, M.D., \\ and A. Leland Albright, M.D.
}

BethanyKids at Kijabe Hospital, Kijabe, Kenya

\begin{abstract}
Object. Ventriculoperitoneal (VP) shunts in Sub-Saharan Africa are traditionally associated with high complication rates and poor outcomes. The aim of this study was to review one large institutional experience with VP shunts, to evaluate the feasibility of shunt insertion procedures with acceptable long-term outcomes in Africa, and to identify factors correlated with good and/or poor outcomes.

Methods. A retrospective study was conducted by reviewing the charts of all children who underwent primary (93\%) or subsequent VP shunt insertions at the Kijabe Hospital between November 2004 and March 2007. Epidemiological data, clinical investigations, etiology of the hydrocephalus, details of the VP shunt insertion, outcome at follow-up, and morbidity and mortality data were collected. Outcomes were graded as good, fair, or poor, according to visual, motor, and seizure criteria.

Results. The authors analyzed 593 VP shunt insertions in 574 patients. The sex distribution was $53 \%$ male and $47 \%$ female. The mean age at shunt insertion was 8.5 months (range 0-309 months). The commonest etiologies for hydrocephalus were spina bifida $(43.4 \%)$ and postinfectious $(27.7 \%)$. Follow-up was available in $76 \%$ of children, with a mean follow-up period of 8.9 months (range 2-30.5 months). The median patient age was 3.3 months. The overall shunt function rate at 2 years was $65 \%$, and the complication rate per procedure was $20 \%$, with infection encountered in $9.1 \%$ and shunt malfunction in $11 \%$. Complications were significantly related to hydrocephalus etiology and to sex $(p=0.03$ and $p=0.01$, respectively). Overall outcomes were good in $40.2 \%$ and poor in $59.8 \%$. Overall mortality in the group was $7.1 \%$. Younger patients who survived had an overall good outcome $(\mathrm{p}=0.0001)$. Only $10 \%$ of patients with a head circumference greater than $60 \mathrm{~cm}$ had a good outcome.

Conclusions. Despite limited resources, VP shunt procedures can be carried out in Sub-Saharan Africa with acceptable complication rates and fair long-term outcomes. (DOI: 10.3171/2010.7.PEDSO9543)
\end{abstract}

KEY WoRds • ventriculoperitoneal shunt • hydrocephalus • Chhabra shunt

$\mathrm{H}$ YDROCEPHALUS is the most commonly treated neurosurgical condition in pediatric practice..$^{14}$ While hydrocephalus has been reported as being more frequent in the developing world, its prevalence there is yet to be determined. ${ }^{29}$ Increased prevalence in Africa is postulated to be due to higher birth rates and consequently higher occurrence of congenital anomalies, as well as higher rates of neonatal infections. ${ }^{29}$ Despite its significant complication rate, VP shunt insertion remains the mainstay treatment for the management of hydrocephalus. Currently, worldwide complication rates range

Abbreviations used in this paper: $\mathrm{BKKH}=$ BethanyKids at Kijabe Hospital; ETV = endoscopic third ventriculostomy; NPIHC $=$ non-postinfectious hydrocephalus; $\mathrm{PIHC}=$ postinfectious hydrocephalus; $\mathrm{QOL}=$ quality of life; $\mathrm{VP}=$ ventriculoperitoneal; $\mathrm{WBC}=$ white blood cell. widely from $1 \%$ to $40 \%,{ }^{6,10,23,30}$ with the mean shunt survival being about 5 years. ${ }^{6}$ Infection is a frequent complication of VP shunts, with reported rates varying from $1 \%$ to $41 \%$, with an average of $10 \%-15 \% .{ }^{13}$

Limited resources in the developing world pose a considerable challenge to the adequate management of hydrocephalus. With available commercial shunts frequently costing hundreds of US dollars, prohibitive prices have further limited their use in this setting. However, the development of inexpensive shunt systems like the Harare and Chhabra shunts has since overcome this barrier, and VP shunt insertion is now commonly performed in SubSaharan Africa. Surgiwear Chhabra shunts are graciously provided free of charge to qualifying centers by the International Society for Spina Bifida and Hydrocephalus, Brussels, Belgium (www.ifglobal.org).

Not surprisingly, reported complication rates in Sub- 
Saharan Africa are significantly higher than those in Western literature. ${ }^{15,17}$ A limited number of studies have explored outcome following VP shunt insertion in this resource-poor setting. A recent study from Zimbabwe revealed a medium-term survival (over 2 years) of only $33 \%-47 \%$ of patients, of whom $46 \%$ suffered from intellectual impairment. ${ }^{17}$ In light of such poor results, that author questioned the justification of providing universal treatment to all hydrocephalic children presenting in a setting plagued by strained economic resources.

The current study reviews rates of complications after VP shunt insertion in hydrocephalic patients treated over a 29-month period in a specialized surgical unit of a Kenyan hospital. The data collected were used to analyze risk factors for complications and to evaluate outcome after VP shunt insertion, aiming to determine outcome predictors following VP shunt insertion in hydrocephalic children.

\section{Methods}

We performed a chart review of all patients who underwent VP shunt insertion at BKKH in Kijabe, Kenya, between November 2004 and March 2007. Patient records were obtained from a computerized database and complemented by individual chart information. Data collected included patient demographics, clinical investigations, etiology of the hydrocephalus, details of the VP shunt insertion procedure, outcome at follow-up, morbidity, and mortality.

\section{Investigations}

Each patient who presented to BKKH with the suspected diagnosis of hydrocephalus underwent cranial ultrasonography. Ventricular size was measured across the frontal horns of the lateral ventricles in the axial projection. Computed tomography scanning reports were available only in $3 \%$ of the patients. A ventricular tap was routinely carried out for CSF WBC count, red blood cell count, glucose, and protein prior to surgery. Gram stain analysis and cultures were also obtained for all patients who presented with a CSF WBC greater than 10 cells/ $\mathrm{ml}$. A low threshold for suspicion of shunt infection was adopted in an effort to minimize missed CSF infections prior to VP shunt insertion.

\section{Origin of Hydrocephalus}

Based on specific details of the patient history, ultrasonography, and CT scanning reports, and to a lesser extent CSF parameters, the etiology of the hydrocephalus was postulated. Etiologies were classified into 4 main categories: PIHC, NPIHC, spina bifida, and prematurity.

The category PIHC was used in cases in which one of the following criteria were met: 1) There was a clear history of meningitis, which was followed by onset of the hydrocephalus. 2) There was a history of a febrile illness, followed in closely by the onset of hydrocephalus, with no symptoms of increased intracranial pressure noted before the advent of the febrile illness. 3) Ultrasonography and CT scans demonstrated postinfectious sequelae such as loculation in the ventricles. In a few recent cases, ventriculoscopy findings were also used to support the diagnosis of PIHC.
The category of NPIHC included cases of congenital hydrocephalus due to aqueductal stenosis, those associated with posterior fossa cysts (Dandy-Walker malformation) and other congenital malformations, and hydrocephalus associated with tumors.

\section{Ventriculoperitoneal Shunt Insertion}

The VP shunt was inserted only if the CSF WBC count was less than 10 cells $/ \mathrm{ml}$, the patient was afebrile, acceptably nourished, and had a hemoglobin level higher than $9 \mathrm{~g} / \mathrm{dl}$. All patients who presented with spina bifida underwent a routine defect closure, and the VP shunt was typically inserted 1 week later, allowing for initial healing of the back wound. Surgical procedures were carried out by 2 consultant pediatric/rehabilitative surgeons and by several visiting neurosurgeons. The Chhabra medium pressure "slit-valve" shunt was used in all primary VP shunt insertions. By default, the ventricular catheters were inserted in a right parietooccipital location; alternative sites were used in cases of skin erosion or excoriation, in cases in which gross asymmetry of the ventricles was demonstrated on ultrasonography, or during secondary insertions. Intravenous cefuroxime was given preoperatively, and intrathecal vancomycin was administered during the operative procedure.

Follow-up was routinely conducted every 2 months for the first 6 months and every 4 months thereafter; beyond 2 years after VP shunt insertion, the patients were seen twice yearly. Most follow-up visits occurred in mobile clinics run by BKKH in 15 sites across Kenya; immediate referral to BKKH was made on any suspicion of a clinical complication.

Parameters of head circumference, vision, motor activity, and seizures were recorded on standardized sheets at each follow-up visit and filed in the patients' notes. Vision and motor activity were assessed using simple clinical rating scales. For analytical purposes, these parameters were recoded into motor and visual outcomes. A "good" overall outcome was defined in children as normal vision, normal motor activity (for age), and no seizures.

\section{Shunt Complications}

Shunt complications were classified as either mechanical or infectious. A diagnosis of shunt infection was made when positive Gram stains or cultures of a pathogenic organism were obtained from a ventricular tap or lumbar puncture. Additional features included high-grade fever $\left(>38.5^{\circ}\right)$, symptoms of shunt malfunction, and abdominal pain or distention. In the absence of positive cultures or Gram stains, a CSF WBC count greater than 50 cells $/ \mathrm{ml}$ in addition to any of the aforementioned features was deemed diagnostic.

All infectious complications were managed by removal of the VP shunt, followed by administration of antibiotics. External ventricular drainage to aid decompression, plus irrigation and administration of intrathecal antibiotics was used in the following 3 situations: infection accompanied by shunt malfunction, gross pus within the ventricular system, and when conservative management (that is, VP shunt removal) was followed by worsening signs of increased intracranial pressure. 


\section{Outcomes of VP shunt insertion in Sub-Saharan Africa}

The external ventricular drain used was the ventricular catheter of a Chhabra shunt. A new VP shunt was only inserted after the CSF WBC count decreased to lower than 50 cells $/ \mathrm{ml}$ and additional cultures were negative.

\section{Statistical Analysis}

Data analysis was performed using SPSS. Parametric means were compared using Student t-test; nonparametric statistics included the Pearson chi-square and the Mann-Whitney test. Statistical significance was set at $\mathrm{p}$ $<0.05$.

\section{Results}

Records for 574 patients who underwent VP shunt insertion at BKKH between November 2004 and March 2007 were analyzed. A slight majority (53\%) of the patients were male. Ages ranged from 1 day to 25 years, with a mean age at presentation of 8.5 months and a median age of 3.3 months; $84.6 \%$ of the children were younger than 1 year old. Figure 1 illustrates the various etiologies of hydrocephalus in our population, while Table 1 shows the age at presentation by etiology. The mean head circumference at presentation was $46.5 \pm 8.4 \mathrm{~cm}$ (90th percentile), and the mean ventricular size was 43 $\pm 14.5 \mathrm{~mm}$. Computed tomography scans were available for 17 patients, and they aided in the diagnosis of DandyWalker malformations in 2 children and intrahemispheric abscesses in another 2 .

Forty-seven CSF samples obtained before shunt placement (9\%) had a WBC count greater than 10 cells/ $\mathrm{ml}$. Among these, positive bacterial culture results were obtained in 6 patients: gram-negative rods in 2 cases (one nonlactose fermenting, possibly Salmonella spp.) and gram-positive cocci in 4 (2 Streptococcus spp. and 2 coagulase-negative Staphylococcus spp.).

\section{Ventriculoperitoneal Shunt Insertion}

Chart notes on a total of 593 VP shunt insertion procedures were analyzed. Of these, $551(93 \%)$ were primary VP shunt insertions at BKKH, $23(4 \%)$ were secondary insertions in patients who had undergone the original VP

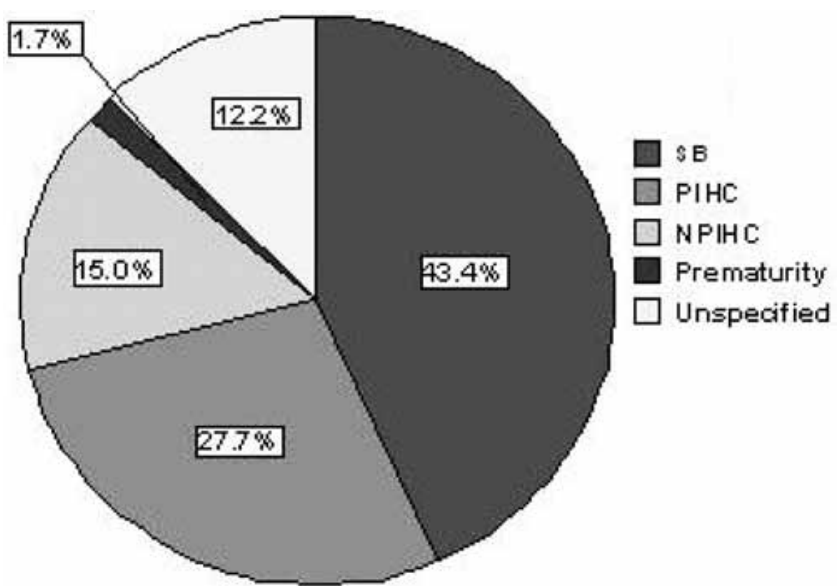

Fig. 1. Pie chart showing the various etiologies of hydrocephalus in our population. SB = spina bifida.
TABLE 1: Percentage of the various etiologies of hydrocephalus within 5 age groups

\begin{tabular}{cccccc}
\hline & \multicolumn{5}{c}{ Etiology (\%) } \\
\cline { 2 - 6 } Age (mos) & Spina Bifida & PIHC & NPIHC & Prematurity & Unknown \\
\hline$\leq 3.0$ & 66.2 & 10.1 & 13.7 & 0.4 & 9.7 \\
$3.1-6.0$ & 27.3 & 37.4 & 13.1 & 5.1 & 17.2 \\
$6.1-9.0$ & 14.1 & 53.1 & 17.2 & 3.1 & 12.5 \\
$9.1-12.0$ & 22.2 & 48.9 & 17.8 & 2.2 & 8.9 \\
$12.1+$ & 21.6 & 43.2 & 18.2 & 1.1 & 15.9 \\
\hline
\end{tabular}

shunt inserted at another institutions, and $19(3 \%)$ were secondary insertions following complications of primary VP shunt insertions originally inserted at BKKH. The median time delay from diagnosis of hydrocephalus to the first VP shunt insertion at BKKH was 6 days (mean $10 \pm 17$ days). For patients without spina bifida and with clear CSF, the median delay was 2 days (mean 6 days). Three-quarters of all VP shunt insertion procedures were performed by the 2 main surgeons at the center, and the remaining procedures were performed by visiting neurosurgeons. The shunt function rate at 2 years was $65 \%$ (Fig. 2).

\section{Complications}

No intraoperative complications were noted. Early complications were recorded in 4 patients $(0.7 \%)$ : 2 suffered cardiorespiratory arrests and died on postoperative Days 2 and 4 after uneventful VP shunt insertion procedures. Another 2 patients had aspiration pneumonia, with 1 fatal outcome. No significant difference was noted between complication rates of the main surgeons and the visiting neurosurgeons. Data on the complication rates among the various surgeons are summarized in Table 2 .

Follow-up was obtained in 434 patients (76\%) with a mean follow-up period of $8.9 \pm 6.6$ months (range 2-31 months). A total of 119 complications (20.0\%) occurred in 104 patients within the study period (Table 3 ). The average time from shunt insertion to the first complication was $3.5 \pm 4.1$ months (range $0.1-18$ months). The vast majority of these complications (78\%) occurred within the first 6 months after VP shunt insertion, another $15.1 \%$ occurred between 6-12 months, and 6.7\% during the 2nd year.

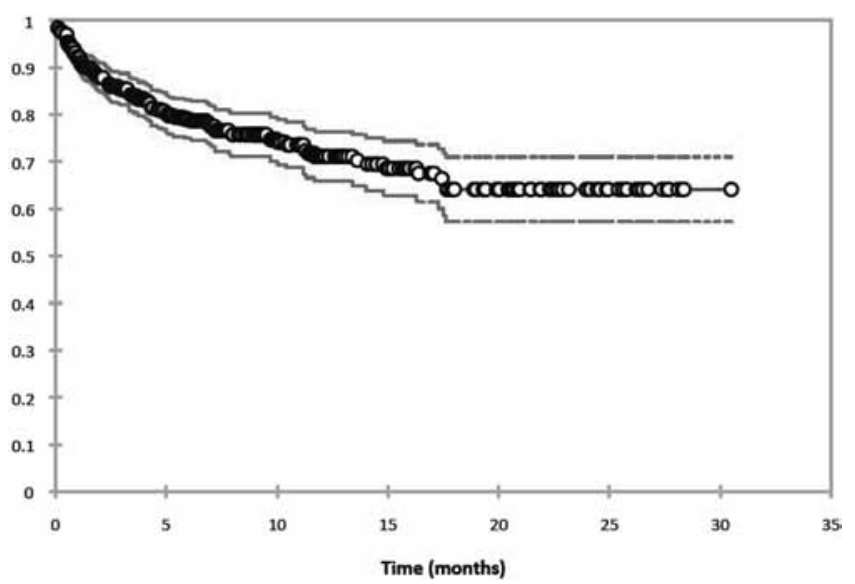

FIG. 2. Survival distribution function in months. 
TABLE 2: Complication rates among the various surgeons*

\begin{tabular}{ccccc}
\hline & & \multicolumn{3}{c}{ No. of Complications (\%) } \\
\cline { 3 - 5 } Surgeon No. & No. of Cases & Mechanical & Infectious & Mixed \\
\hline 1 & 262 & $27(10.3)$ & $28(10.7)$ & $7(2.7)$ \\
2 & 120 & $15(12.5)$ & $6(5.0)$ & $4(3.3)$ \\
3 & 131 & $22(16.8)$ & $7(5.3)$ & $1(0.8)$ \\
\hline
\end{tabular}

* Surgeons 1 and 2 are the main operating surgeons at BKKH performing VP shunt insertions for the cases that were followed up during this period study. Surgeon 3 represents visiting neurosurgeons who performed the VP shunt insertions during this period of study.

The numbers and percentages of various complication types are listed in Table 3 . Sex was significantly correlated to the occurrence of complications $(\mathrm{p}=0.01)$, and especially of infectious complications $(\mathrm{p}=0.009)$, with girls being more prone to VP shunt complications. On the other hand, the etiology of the hydrocephalus was correlated to mechanical complications: patients with spina bifida were significantly more likely to develop mechanical complications $(\mathrm{p}=0.03)$ than those with isolated hydrocephalus.

The visual outcome of the children correlated significantly with age (older patients had a worse outcome, $\mathrm{p}=0.004)$, and with ventricular size at presentation $(\mathrm{p}<$ $0.001)$. There was no significant improvement in vision after VP shunt insertion. Motor outcome, on the other hand, was inversely related with age (younger patients had a worse outcome, $\mathrm{p}<0.0001$ ), with hydrocephalus etiology (patients with spina bifida naturally had a poor motor outcome, $\mathrm{p}<0.0001$ ), and with history of seizures at presentation $(\mathrm{p}<0.02)$. Seizure activity postoperatively was associated with a PIHC etiology $(p=0.002)$ and with a previous history of seizures $(\mathrm{p}=0.016)$.

There were 37 deaths recorded in our series $(8.5 \%$ of patients who underwent follow-up), although the mortality may be higher in patients lost during follow-up. In the mortality group, the mean interval to death was 5 months (median 2 months, range 0.1-24 months). Only 5 deaths (1\%) were clearly related to shunt complications. Another 5 children died of pneumonia and 1 of malaria. The cause of death in 26 cases was not known. Death correlated with age (younger children were more likely to die, $\mathrm{p}=$

TABLE 3: Distribution of the various complications among the various age groups

\begin{tabular}{llllc}
\hline & \multicolumn{3}{c}{ Age Group (\%) } & \\
\cline { 2 - 4 } Complication & $0-6$ Mos & $6-12$ Mos & 12+ Mos & $\begin{array}{c}\text { Total No. of } \\
\text { Complications }(\%) \dagger\end{array}$ \\
\hline mechanical & $49(41.0)$ & $12(10.1)$ & $4(3.4)$ & $65(11)$ \\
infectious & $33(27.7)$ & $6(5.0)$ & $3(2.5)$ & $42(7)$ \\
mixed & $11(9.2)$ & 0 & $1(0.8)$ & $12(2)$ \\
total & $93(78.2)$ & $18(15.1)$ & $8(6.7)$ & $119(20)$ \\
\hline
\end{tabular}

* The percentage is calculated as the distribution of complications among the various age groups.

$\dagger$ The percentage is calculated as the distribution per total number of VP shunt insertions (593 insertions).
0.001 ), with the etiology of hydrocephalus (death rate was significantly higher among patients with spina bifida, $\mathrm{p}=$ $0.015)$, and with presence of spasticity at presentation ( $p$ $=0.026$ ).

Younger patients had a worse overall outcome $(\mathrm{p}<$ 0.0001). A significant inverse relationship was observed between head circumference and overall good outcomes ( $p<0.0001)$ (Fig. 3). Patients with a head circumference greater than $60 \mathrm{~cm}$ had only a $10 \%$ chance of having a good outcome after VP shunt insertion. Hydrocephalus etiology was also significantly related to good outcome ( $p$ $<0.0001)$. Patients with spina bifida had a poorer outcome (because of generally poor motor outcome).

\section{Discussion}

Despite the increasing use of endoscopic procedures, VP shunt placement remains the principal method of treating hydrocephalus, especially in developing countries. It is the gold standard against which newer procedures are judged. Despite significant innovation in shunt design and the operative environment over the past decades, VP shunts are still associated with substantial complication rates. Worldwide failure rates currently still range between $25 \%$ and $40 \%$ within the 1st year following insertion.

In our study, the overall complication rate of VP shunt insertion (at a mean follow-up of 9 months) was $20 \%$. The infectious complication rate was $9.1 \%$, which is comparable to the $9.7 \%$ rate reported in the standard-setting recent large-scale Ugandan study, and encouragingly lower than the 20\%-50\% range generally reported in most studies from Sub-Saharan Africa and other developing countries over the past decade..$^{12,15,16,18,20,21,25}$ In fact, our rate of infectious complications lies just outside the $2 \%-9 \%$ range of infectious complications currently reported in North America. ${ }^{6}$ The overall rate of pure mechanical failure (that is, not complicated by infection) was $11 \%$. Few studies have addressed the rate of mechanical failures only, but a randomized multicenter study from Canada reported a combined $40 \%$ failure rate within the 1st year of followup..$^{10}$ This places our $11 \%$ mechanical failure at a 9-month mean follow-up well within Western data.

The calculated mortality rate in this study was $6 \%$, although the true figure was probably higher considering the likelihood of death in patients lost to follow-up. There was

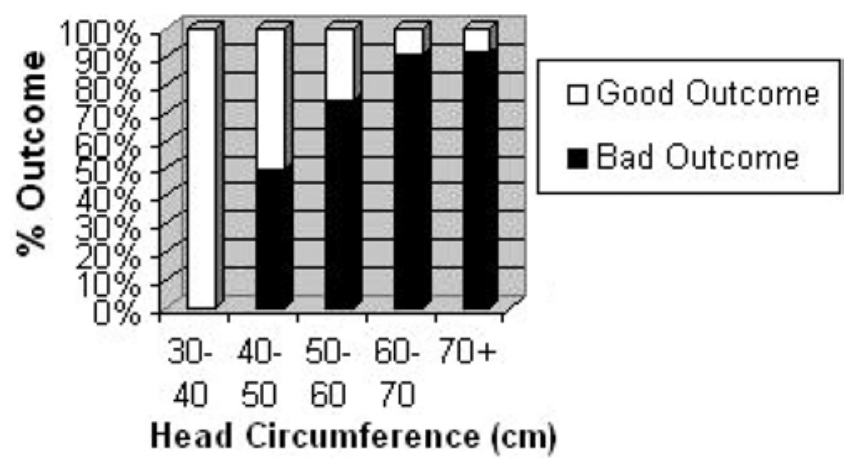

Fig. 3. Bar graph showing the percentage of good outcome by head circumference. 


\section{Outcomes of VP shunt insertion in Sub-Saharan Africa}

a significant relationship between mortality rate and age at diagnosis; younger children were more likely to die. There was also a significant association between mortality rate and presence of spina bifida. These findings are not supported by the existing literature and may reflect the higher mortality in our setting of neonates with spina bifida-related hydrocephalus, who typically undergo surgery in the 1st month of life. While our anesthesia and perioperative care are acceptable for our setting, neonatal surgery remains a challenge because of concurrent infant malnutrition, nursing care, and the difficulty in diagnosing and treating Chiari symptoms in neonates.

Besides morbidity and mortality, the other overall outcome indicator is QOL. In the absence of good QOL data, "good outcome" was used as a surrogate marker of QOL in our study. Owing to the retrospective nature of our study and the limited follow-up data, "good outcome" was defined as normal vision and motor function together with absence of seizures on follow-up. Naturally, this variable was significantly confounded by the frequent motor impairment of children with hydrocephalus due to spina bifida.

\section{Patient Volumes}

The average yearly number of shunt-related procedures was 260 during the study period, and has surpassed 500 since 2007 . By comparison, yearly volumes in the regional literature ranged from 34 to $50 . .^{15,18,20}$

\section{Predictors of Outcome}

Sex. Fifty-three percent of the patients were male in our study, which is consistent with a previously reported male preponderance in hydrocephalus. ${ }^{2,20}$ Our results also showed a significant relationship between sex and complications, with female patients more likely to develop infectious complications. This relationship has not been previously documented in relation to CSF shunt infections, ${ }^{7,8,22}$ and it deserves further exploration. Social and environmental factors may account for this difference. In this setting, higher value is placed on the boy child, leading to better care provided by the parents in the postoperative period. Biological differences between the sexes may also play a significant role. Emerging studies have found a significant difference in early HIV infection between the sexes in Sub-Saharan Africa where female patients have been shown to be more prone to acquiring early infection. ${ }^{26}$ Genetic factors leading to possible immunological differences between the sex have been postulated.

Age. Reports have suggested that young age is a risk factor for VP shunt complications, and in particular infectious complications. ${ }^{11}$ This has been attributed to immunological immaturity early in life, caused by declining levels of maternal IgG antibodies coupled with less efficient complement activity and cellular immunity. ${ }^{11}$ The current study has not corroborated this finding, possibly because of the narrow age range of our patients. Di Rocco et al. ${ }^{9}$ found a significant difference in complication rates between children older and younger than 2 years (the vast majority [84.7\%] of the patients in our study were younger than 1 year of age). On the other hand, we have found age to significantly influence visual outcome, motor outcome, overall good outcome, and death. Older patients tended indeed to have better outcomes in all these variables. It must be noted, however, that the cause of hydrocephalus was an important confounding variable; spina bifida, with its attendant poorer motor outcome, was commoner in the younger patient population.

Delay in presentation is a common feature in patients presenting with hydrocephalus in the developing world, ${ }^{29}$ and it is closely related to several socioeconomic factors. ${ }^{2}$ Parents may fail to recognize the importance of a rapidly increasing head size (education), lack funds to attend regular postnatal visits where an early diagnosis of hydrocephalus may be established (resources), or live too far from health care points (access).

Comorbidity. Comorbidities were present in $16 \%$ of our patients. Congenital talipes equinovarus was the commonest condition, followed by malnutrition. Malnutrition has an obvious impact on VP shunt outcome because of immune depression, thin skin, and poor healing. While an attempt was made in isolated cases to provide nutritional care before shunt placement, most children with hydrocephalus are probably at least undernourished because of the cycle of poverty, maternal malnutrition, and neurological impairment. Respiratory tract infections and malaria, other important causes of infant mortality in Africa, were also present in our series.

Head Circumference. A key finding of this study was that head circumference was strongly related to outcome. Patients with a head circumference larger than $60 \mathrm{~cm}$ had, regardless of age, only a $10 \%$ chance of a good outcome following VP shunt insertion. Although interventional studies evaluating the outcome of shunt procedures in the developing world are absent, it is commonly believed that outcome in terms of physical and intellectual function is widely determined by the extent of disease at the time of presentation. Given the significant rates of shunt complications in shunt-treated patients in the developing world as well as access issues in managing late complications, committing children with poor chances of good outcome to shunt dependency is hard to justify.

Interestingly, overall mortality rates were inversely related to head circumference. This was most likely due to the confounding effect of etiology. Patients with spina bifida, whose mortality was higher than the rest, typically presented at an earlier age and therefore had a smaller head circumference.

Imaging Investigations. Ultrasonography is often the only paraclinical option for hydrocephalus diagnosis in the developing world. Computed tomography scans are limited to urban settings and are expensive. As a result, shunt placement is often carried out in instances in which the etiology has not been fully clarified, as exemplified by the patient with an unrecognized Dandy-Walker cyst. Other diagnoses potentially missed without adequate imaging include tumors, hydranencephaly, and extracerebral fluid collections-all potentially adversely affecting the final patient outcome. 
Etiology. While congenital hydrocephalus in term infants and intraventricular hemorrhage in preterm infants are the most common etiologies of hydrocephalus in developed countries, it has been purported that PIHC is the most common etiology of hydrocephalus in East Africa $^{1}$ and in India as well. ${ }^{28}$ In our study the most common etiology of hydrocephalus was spina bifida, accounting for $44 \%$ of the cases. Postinfectious hydrocephalus accounted for $28 \%$ of the cases, being the second commonest etiology. Our findings probably reflect patient referral patterns, as our center treats an unusually large number of children with spina bifida, the vast majority of whom require shunt placement. ${ }^{29}$

Spina bifida has been associated with increased rates of VP shunt complications, especially infections..$^{10}$ This risk is mainly attributed to bacterial contamination via the open neural tube defect and its attendant risk of CSF infection. Patients in our setting commonly present late with open, leaking, and grossly infected wounds. This has influenced our management of hydrocephalus associated with spina bifida. Shunt insertion is usually done 6-7 days after closure of the spina bifida defect, once the wound is healing without evidence of infection or breakdown.

\section{Ventriculoperitoneal Shunt Insertion}

There is support in the literature for the importance of intraoperative factors (such as double-gloving) in the prevention of shunt complications. ${ }^{4,27}$ It is indeed possible to significantly reduce the VP shunt complication rates by strict adherence to surgical asepsis principles, ${ }^{24}$ leading to infection rates as low as $1 \% .^{3,23}$ Some case-control studies have reported significant reduction in complication rates following modification of surgical protocols, while in other studies the evidence has been largely inconclusive. ${ }^{19}$ Some studies have reported a difference in complication rates between experienced and less experienced surgeons. ${ }^{5}$ The fact that surgeons and neurosurgeons had similar outcomes in this study has implications for the treatment of pediatric hydrocephalus in developing countries, which have few neurosurgeons.

In this study, we are reporting acceptable complication rates (9\% infection and $11 \%$ mechanical failure rates) in a limited-resource environment traditionally prone to high complication rates. We have achieved this through standardized insertion techniques focused on avoiding shunt manipulation and shortening the operative procedure, close attention to aseptic techniques, and protocolbased postoperative care provided by trained staff in a dedicated center specialized in the treatment of children with neurological disabilities. The future of hydrocephalus management in the developing world may well lie in similar high-volume dedicated centers. The volume of hydrocephalic patients treated yearly is a key difference between this study and previous studies reported from Sub-Saharan Africa.

Given the significant complication rates of VP shunts, ETV presents an attractive option in the management of hydrocephalus, and early indications of its application in the developing world setting are promising. ${ }^{29}$ Shunt independence in this setting potentially improves the QOL for patients who may otherwise suffer from multiple shunt complications. A recent study in Uganda found that ETV with choroid plexus cauterization was successful in $70 \%$ of cases of PIHC in children younger than 1 year of age, although the results for younger children with other causes of hydrocephalus were less favorable. ${ }^{29}$ The use of ETV in the developing world is also limited by expensive equipment and a paucity of available expertise. Our institution has recently started to use this procedure, and we hope to have comparative studies between the 2 methods in the near future.

Shunts, on the other hand, are widely available and effective in the management of hydrocephalus irrespective of etiology. Given the availability of reasonably priced or even fully subsidized VP shunts and the fact that VP shunt insertion is a relatively straightforward surgical procedure, shunts will continue to play a pivotal role in the management of hydrocephalus in Africa into the foreseeable future. The $65 \%$ shunt function rate at 2 years is comparable to several Western studies and to ETV results.

\section{Limitations of the Study}

Despite concerted efforts to follow up our patients, $17 \%$ of the patients were lost to follow-up, thereby introducing a significant sampling bias. The lost patients may be presumed to be either dead or they did not experience any complications and had a good outcome. Due to poverty and limited access to care, it was common to have patients present for follow-up only when they had developed complications, rather than regularly as advised.

Our definition of "good outcome" as the absence of seizures, motor difficulties, or visual problems is at best an assumption, since intellectual development and QOL include multiple other unmeasured factors. Our followup was short and middle term. The long-term follow-up results will require several more years of data collection, as it is known that VP shunts have a long-term yearly loss in function.

Finally, the definitive way to reduce the morbidity associated with VP shunts is to prevent the development of hydrocephalus in the first place. Prompt recognition and management of neonatal meningitis (often misdiagnosed as malaria) in this setting is paramount if there is to be a marked reduction in the incidence of hydrocephalus. The other significant cause of hydrocephalus in our study, spina bifida, can be reduced in incidence through folic acid supplementation to all women of childbearing age and through widespread food fortification. Besides primary prevention, secondary prevention of hydrocephalus complications requires public and health care provider education, allowing the prompt recognition and referral of hydrocephalus in its early phases. Such measures would not only decrease the morbidity of shunt placement but also decrease the life-long neurological impairment of children following shunt treatment.

Despite the mainstay use of VP shunts in the management of hydrocephalus in Africa, ethical controversies and challenges remain, especially in the care of children with massive hydrocephalus (head circumference $>60$ $\mathrm{cm})$. Our study confirms the clinical impression of medical futility in the surgical treatment of such children, although parental insistence and the agony of children with "bar- 
rel heads" make nontreatment a difficult option. In some selected cases, a VP shunt may be still the best palliation available.

\section{Conclusions}

Our study has identified the prognostic factors of VP shunts for hydrocephalus in a resource-limited African setting and has documented good outcomes for shunt treatment in this setting, with acceptable complication rates. However, children with a head circumference greater than $60 \mathrm{~cm}$ have only a $10 \%$ chance of a good outcome. Patients who presented with hydrocephalus associated with spina bifida presented at a younger age and had higher mortality rates and higher rates of shunt malfunction; however, following initial survival their outcome was also favorable.

\section{Disclosure}

The authors report no conflict of interest concerning the materials or methods used in this study or the findings specified in this paper.

Author contributions to the study and manuscript preparation include the following. Acquisition of data: all authors. Reviewed final version of the manuscript and approved it for submission: all authors.

\section{Acknowledgments}

The authors thank the International Federation of Spina Bifida and Hydrocephalus, the "neuro team" at BethanyKids at Kijabe Hospital, and Dr. Kim Augustein.

\section{References}

1. Abdullah J, Naing NN: Hydrocephalic children presenting to a Malaysian community-based university hospital over an 8 year period. Padiatr Neurosurg 34:13-19, 2001

2. Adeloye A: Management of infantile hydrocephalus in Central Africa. Trop Doct 31:67-70, 2001

3. Choksey MS, Malik IA: Zero tolerance to shunt infections: can it be achieved? J Neurol Neurosurg Psychiatry 75:8791,2004

4. Choux M, Genitori L, Lang D, Lena G: Shunt implantation: reducing the incidence of shunt infection. J Neurosurg 77: 875-880, 1992

5. Cochrane DD, Kestle JRW: The influence of surgical operative experience on the duration of first ventriculoperitoneal shunt function and infection. Pediatr Neurosurg 38:295-301, 2003

6. Crnich CJ, Safdar N, Maki DG: Infections associated with implanted medical devices, in Finch RG, Greenwood D, Norrby SR, et al (eds): Antibiotic and Chemotherapy: AntiInfective Agents and Their Use in Therapy, ed 8. London: Churchill Livingstone, 2003, pp 575-618

7. Dallacasa P, Dappozzo A, Galassi E, Sandri F, Cocchi G, Masi M: Cerebrospinal fluid shunt infections in infants. Childs Nerv Syst 11:643-649, 1995

8. Davis SE, Levy ML, McComb JG, Masri-Lavine L: Does age or other factors influence the incidence of ventriculoperitoneal shunt infections? Pediatr Neurosurg 30:253-257, 1999

9. Di Rocco C, Massimi L, Tamburrini G: Shunts vs endoscopic third ventriculoscopy in infants: are there different types and/or rates of complications? A review. Childs Nerv Syst 22:1537-1589, 2006

10. Drake JM, Kestle JRW, Tuli S: CSF shunts 50 years on-past, present and future. Childs Nerv Syst 16:800-804, 2000

11. Enger PO, Svendsen F, Wester K: CSF shunt infections in children: experiences from a population-based study. Acta Neurochir (Wien) 145:243-248, 2003
12. Heij HA: The fate of ventriculoperitoneal shunts and outcome of revision surgery. East Central Afr J Surg 5:17-19, 2000

13. Kanev PM, Sheehan JM: Reflections on shunt infection. Pediatr Neurosurg 39:285-290, 2003

14. Kestle JRW, Garton HJL, Drake JM: Treatment of hydrocephalus with shunts, in Albright AL, Pollack IF, Adelson PD (eds): Principles and Practice of Pediatric Neurosurgery. New York: Thieme, 1999, pp 75-90

15. Kinasha ADA, Kahamba JF, Semali IT: Complications of ventriculoperitoneal shunts in children in Dar es Salaam. East Central Afr J Surg 10:55-59, 2005

16. Komolafe EO, Adeolu AA, Komolafe MA: Treatment of cerebrospinal fluid shunting complications in a Nigerian neurosurgery programme. Case illustrations and review. Pediatr Neurosurg 44:36-42, 2008

17. Laurence FL: Treatment of hydrocephalus. East Central Afr J Surg 11:78-80, 2006 (Abstract)

18. Lima MM, Pereira CU, Silva AM: [Ventriculoperitoneal shunt infections in children and adolescents with hydrocephalus.] Arq Neuropsiquiatr 65:118-123, 2007 (Portugese)

19. Mottolese C, Grando J, Convert J, Abdoulrahman M, Lelievre $\mathrm{H}$, Vandenesch F, et al: Zero rate of shunt infection in the first postoperative year in children-dream or reality? Childs Nerv Syst 16:210-212, 2000

20. Mwang'ombe NJM, Omulo T: Ventriculoperitoneal shunt surgery and shunt infections in children with non-tumour hydrocephalus at the Kenyatta National Hospital, Nairobi. East Afr Med J 77:386-390, 2000

21. Oneko M, Lyamuya S, Mhando S: Outcome of hydrocephalus and spina bifida surgery in a referral hospital without neurosurgical services in Tanzania. Eur J Pediatr Surg 12 Suppl 1:S39-S41, 2002

22. Piatt JH Jr, Carlson CV: A search for determinants of cerebrospinal fluid shunt survival: retrospective analysis of a 14year institutional experience. Pediatr Neurosurg 19:233242, 1993

23. Pirotte BJM, Lubansu A, Bruneau M, Loqa C, Van Cutsem N, Brotchi J: Sterile surgical technique for shunt placement reduces the shunt infection rate in children: preliminary analysis of a prospective protocol in 115 consecutive procedures. Childs Nerv Syst 23:1251-1261, 2007

24. Rotim K, Miklic P, Paladino J, Melada A, Marcikic M, Scap $\mathrm{M}$ : Reducing the incidence of infection in pediatric cerebrospinal fluid shunt operations. Childs Nerv Syst 13:584-587, 1997

25. Sibanda EN, Levy LF, Makarawo S: Infection after Harare valve V-P shunt operations: a review of 92 cases. Cent Afr J Med 37:397-403, 1991

26. Taha TE, Nour S, Kumwenda NI, Broadhead RL, Fiscus SA, Kafulafula G, et al: Gender differences in perinatal HIV acquisition among African infants. Pediatrics 115:e167-e172, 2005

27. Thompson DNP, Hartley JC, Hayward RD: Shunt infection: is there a near-miss scenario? J Neurosurg 106 (1 Suppl): $15-19,2007$

28. Udani V, Udani S, Merani R, Bavdekar M: Unrecognised ventriculitis/meningitis presenting as hydrocephalus in infancy. Indian Pediatr 40:870-873, 2003

29. Warf BC: Hydrocephalus in Uganda: the predominance of infectious origin and primary management with endoscopic third ventriculostomy. J Neurosurg 102 (1 Suppl):1-15, 2005

30. Weprin BE, Swift DM: Complications of ventricular shunts. Tech Neurosurg 7:224-242, 2002

Manuscript submitted January 8, 2010.

Accepted July 7, 2010.

Address correspondence to: Dan Poenaru, M.D., BethanyKids at Kijabe Hospital, Box 20, 00220 Kijabe, Kenya. email: dpoenaru@ gmail.com. 